\title{
Retrograde cerebral venous air embolism and the anatomical pathway of air bubbles: a case report
}

\author{
Berrin Erok $^{1}$ (D), Kenan Kıbıcı ${ }^{\text {(D) , Ali Önder Atça }}{ }^{3}$ (D) \\ ${ }^{1}$ Department of Radiology, University of Health Sciences, Prof. Dr. Cemil Taş̧ı̆ğlu City Hospital, İstanbul, Turkey \\ ${ }^{2}$ Department of Neurosurgery, Altmbaş University School of Medicine, Bahçelievler Medical Park Hospital, İstanbul, Turkey \\ ${ }^{3}$ Department of Radiology, Altınbaş University School of Medicine, Bahçelievler Medical Park Hospital, Istanbul, Turkey
}

\begin{abstract}
Pneumocephalus due to cerebral venous air embolism is an uncommon phenomenon. It results from retrograde progression of low weight air bubbles into dural venous sinuses during manipulation of a venous catheter, more frequently a central venous catheter through the subclavian and the jugular veins. However, it may also occur in relation with a peripheral intravenous catheter as in our case. We report a 91 year old female patient with congestive heart failure who had been examined in our emergency department two days previously due to dyspnea and received diuretic treatment through a peripheral intravenous line. She presented with vomiting and headache without obvious neurological deficits. Non-contrast cranial CT scan revealed wide spread punctate air bubbles inside and outside the cranial vault (pneumocephalus), within the venous system. The pneumocephalus was considered as iatrogenic due to the previous peripheral venous catheterization that resulted in retrograde migration of air bubbles through various venous connections into dural venous sinuses and extracranial veins. Since cerebral venous air embolism is a potentially serious complication of various medical procedures, it should be considered in differential diagnosis of nontraumatic headache and vomiting especially when there is a recent manipulation of venous lines. Cranial CT scan is helpful for early diagnosis.
\end{abstract}

Keywords: computed tomography; pneumocephalus; retrograde cerebral venous air embolism

Anatomy 2020;14(3):210-215 @2020 Turkish Society of Anatomy and Clinical Anatomy (TSACA)

\section{Introduction}

Cerebral venous air embolism (CVAE) is a potentially serious complication of various medical procedures performed in almost all clinical specialties such as in neurosurgery during posterior fossa operations, invasive pulmonary procedures (eg. percutaneous lung biopsy) or intravenous (IV) catheterization (central or peripheral). ${ }^{[1-3]}$ The true incidence of CVAE as a result of venous catheterization is unknown since it is usually asymptomatic. Although it has been revealed to develop during manipulations of central venous catheters (CVCs), it may also develop rarely in association with a peripheral IV line. ${ }^{[4,5]}$ It is formed when the air bubbles overcome the gradient between the external atmospheric pressure and the intravenous pressure to enter the blood stream. This concept of retrograde passage of air bubbles was first described in a letter to the editor by Ploner et al ${ }^{[6]}$ in 1991.

Clinical presentation and prognosis in patients with CVAE varies depending on the volume and territory of the air bubbles in the cerebral vasculature. In most of the cases, patients have nonspecific sysmptoms such as headeache, nausea, vomiting, vertigo and confusion. However, it may also be accompanied by more severe neurological findings such as decreased consciousness or seizure. In addition, it can act like a thrombotic emboli and cause ischemic stroke by occluding the end arteries resulting in focal neurological deficits. ${ }^{[5]}$ Therefore, prevention should be the priority by properly positioning the patients and keeping the lumens of venous catheters filled with saline prior to any manipulations. 


\section{Case Report}

A 91-year old female patient with congestive heart failure (CHF) secondary to ischemic heart disease presented to our emergency department with the chief complaint of vomiting and headache. There was no recent head trauma or history of any surgical procedures. She was first examined in our emergency department for shortness of breath two days ago and was discharged with relief of symptoms after receiving diuretic therapy through a peripheral IV line. On neurological examination, the patient was well oriented without obvious neurological deficits. She had undergone a non-contrast cranial computed tomography (CT) in order to exclude intracranial hemorrhage. On CT, the brain parenchyma was normal but there were wide spread small punctate air bubbles inside and outside the cranial vault. The air bubbles were observed to spread into right transverse sinus (Figure 1), the right sigmoid sinus and bilateral cavernous sinuses (Figure 2a), the right inferior petrosal sinus (Figure 2b), cervical epidural venous plexus at the level of foramen magnum (Figure 3a) and at the level of cervical spine (Figure $3 \mathbf{b}$ ), the right vertebral venous plexus (Figure 3b), venous plexus of the right hypoglossal canal (Figure 4a), bilateral ophtalmic veins (Figures $4 b$ and $4 c$ ), the right pterygoid venous plexus
(Figures 3a, 4a and 4d) and the right superficial temporal vein (Figure 2a). When compared with the CT scan performed 2 months ago the air bubbles were found to be a new finding. Concerning the typical distribution of air bubbles throughout the venous vasculature and the lack of any fracture involving the cranial vault or air spaces of the head, the pneumocephalus was considered to happen due to IV injection of air through the peripheral venous catheterization performed two days ago. She was positioned in Trendelenburg position and received $100 \%$ oxygen therapy. During close follow up at the emergency department, no clinical deterioration was observed. A follow up examination was planned in our neurology department and she was discharged with improvement of the complaints.

\section{Discussion}

Pneumocephalus also known as pneumocranium refers to the presence of air or gas within the cranial vault either extraaxial (epidural, subdural, subarachnoid) or intraaxial (parenchymal, intraventricular, intravascular). Localization of the air bubbles to a specific compartment and assessment of the entryway is important in diagnosis and management of the patients in emergency departments.
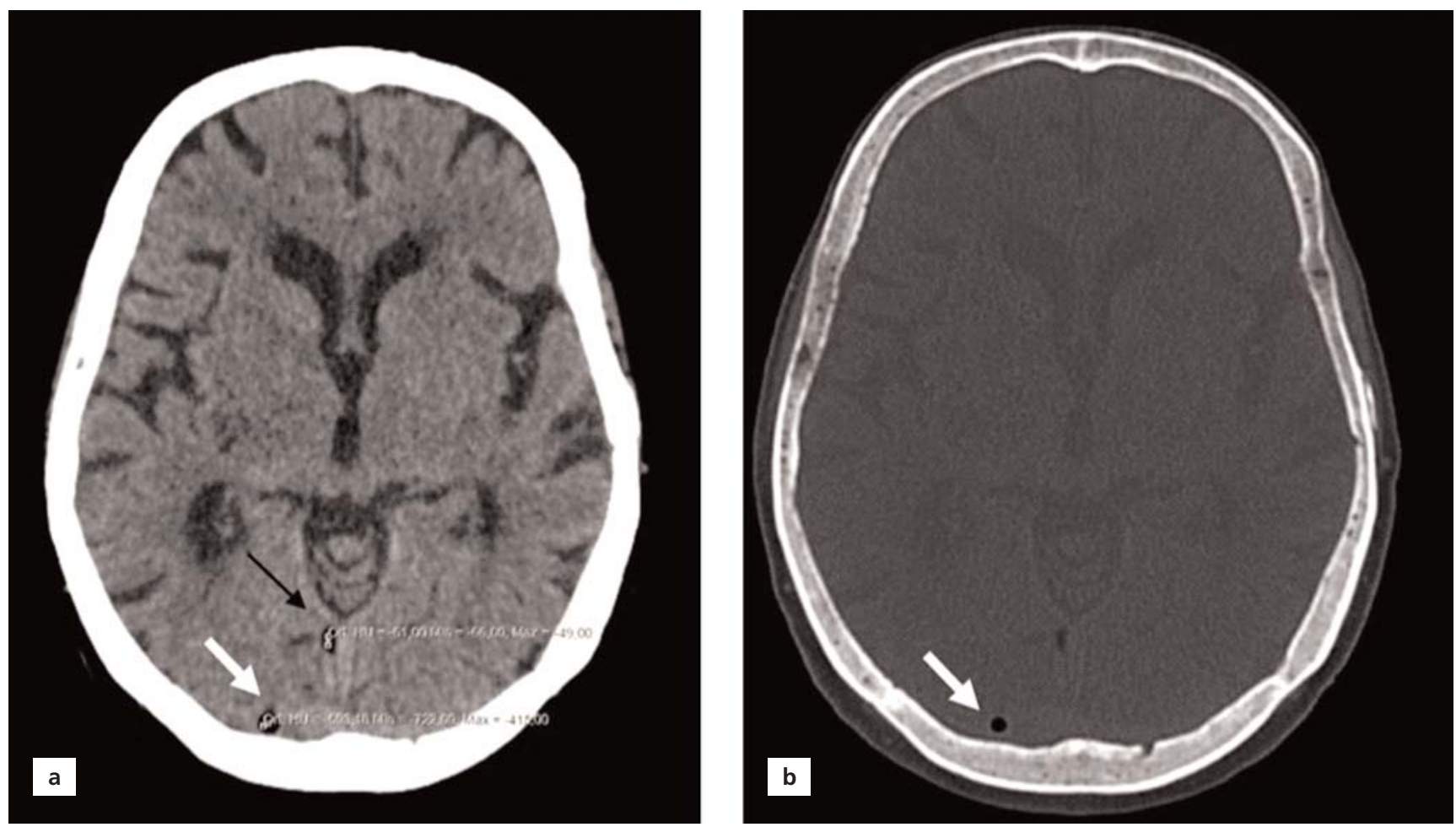

Figure 1. Parenchymal window (a) and bone window (b) images of non-enhanced cranial CT showing air bubbles in the transverse sinus (white arrows). A small midline lipoma is pointed by black arrow. 

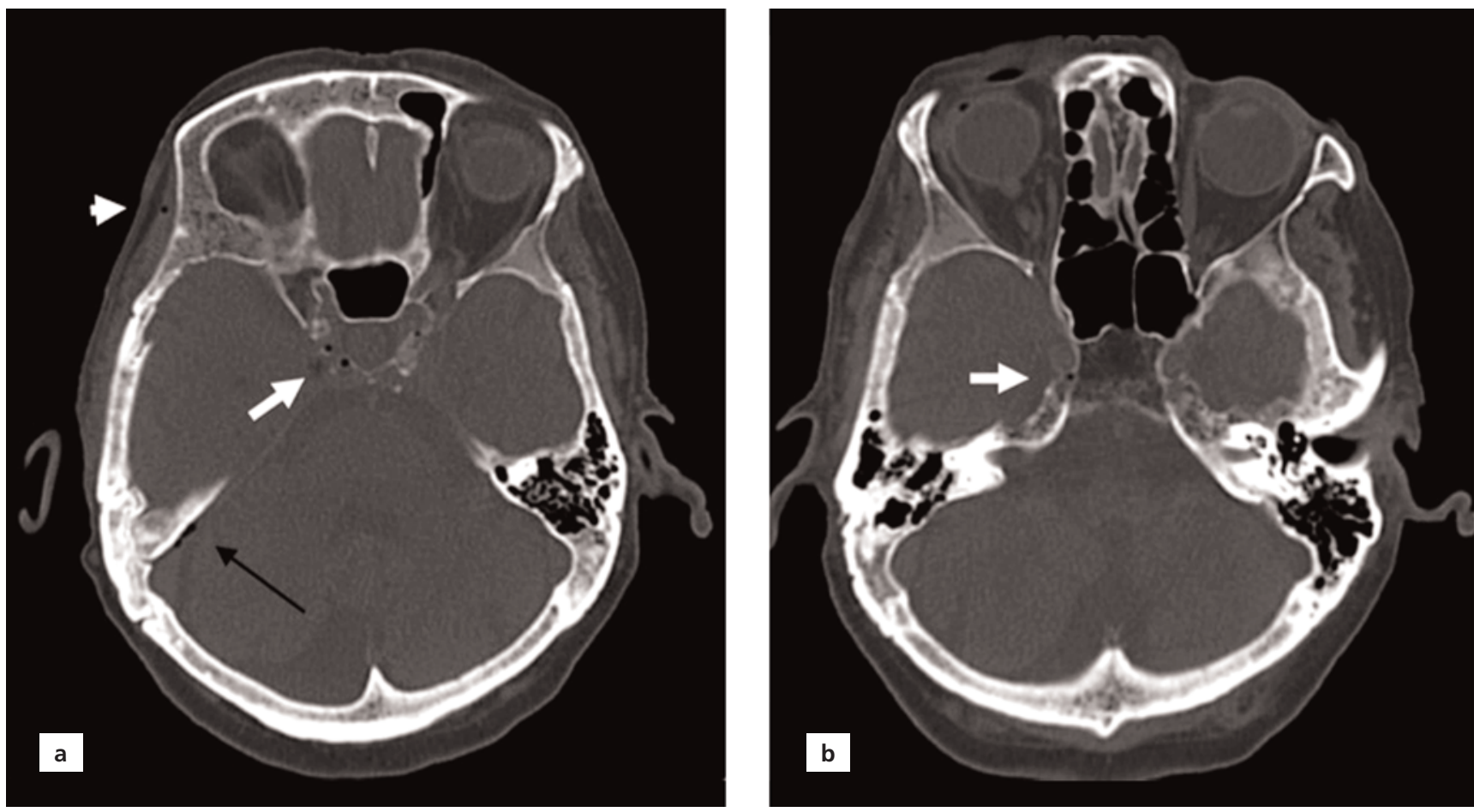

Figure 2. Bone window images of non-enhanced cranial CT. (a) Air bubbles in the cavernous sinus (white arrow), right sigmoid sinus (black arrow) and right superficial temporal vein (short white arrow); (b) air bubbles in the right inferior petrosal sinus (white arrow).
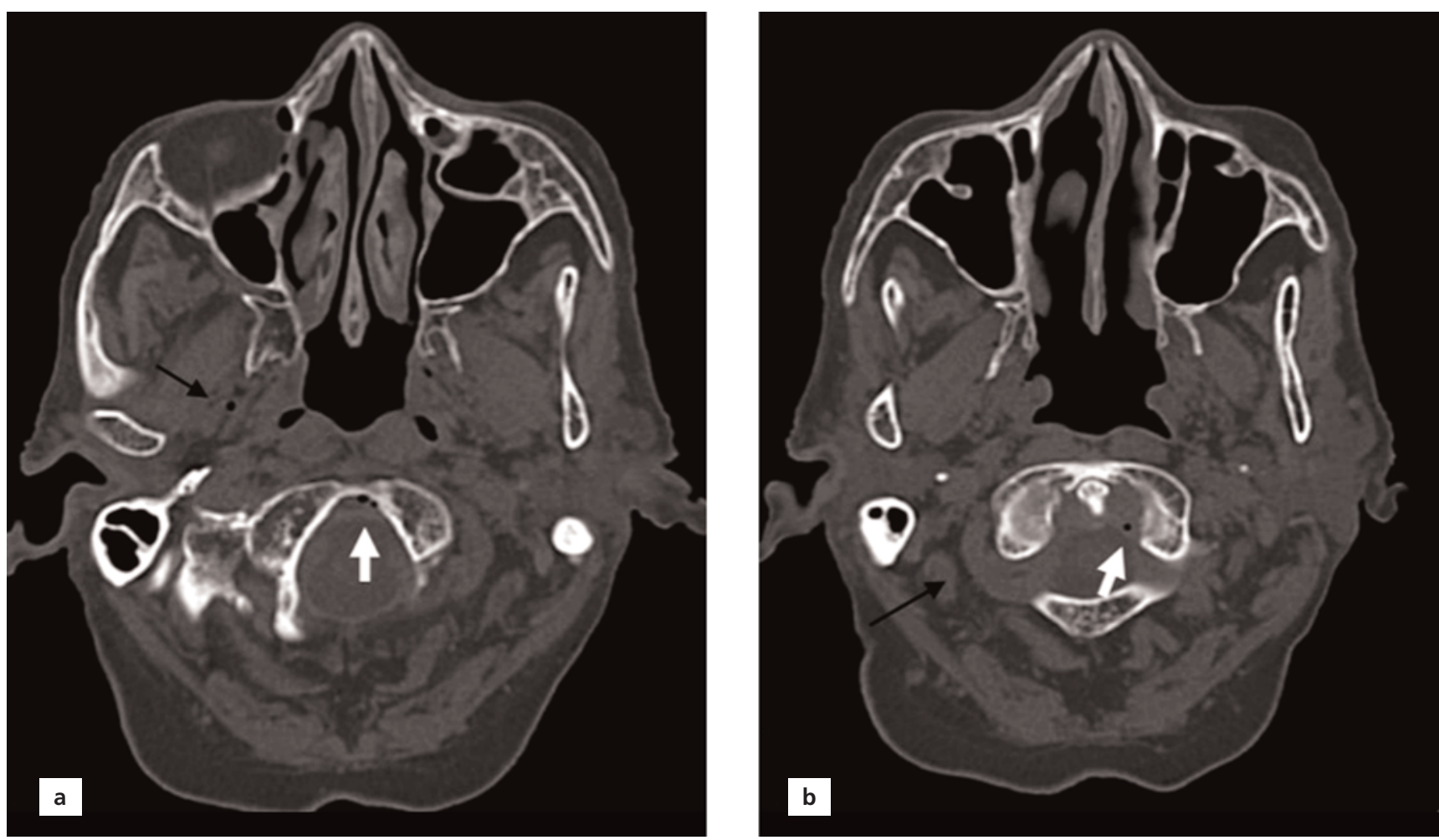

Figure 3. Bone window images of nonenhanced cranial CT. (a) Air bubbles in cervical epidural venous plexus at the level of foramen magnum (white arrow) and in the right pterygoid venous plexus (black arrow); (b) air bubbles in cervical epidural venous plexus at the level of cervical spine (white arrow) and in the right vertebral artery venous plexus (black arrow). 

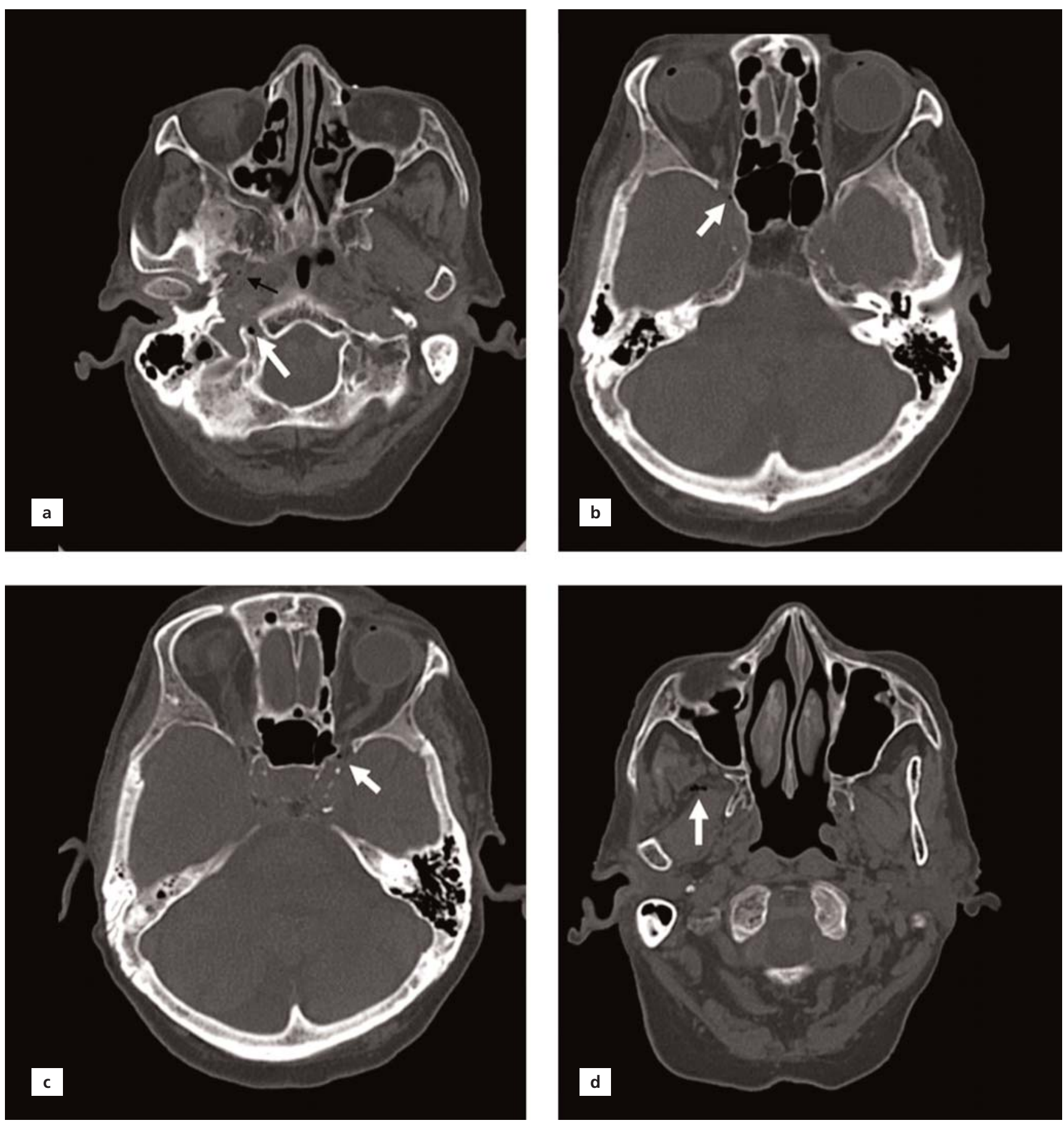

Figure 4. Bone window images of nonenhanced cranial CT. (a) Air bubbles in the venous plexus of the right hypoglossal canal (white arrow) and in the right pterygoid venous plexus (black arrow); (b) air bubble in the right ophtalmic vein (white arrow); (c) air bubble in the left ophtalmic vein (white arrow); (d) air bubbles in the right pterygoid venous plexus (white arrow).

Pneumocephalus associated with CVAE preasumbly due to IV injection of air is an uncommon phenomenon and occurs during the placement or removal of a venous catheter. It mainly develops in association with a central venous catheter rather than a peripheral IV line. In a ret- rospective study, covering 24 months period and analyzing total of 8747 cranial CT scans, intracranial air bubbles presumably due to the injection of air through peripheral IV lines was established in 3 cases with an incidence of $0.034 \%$ (3/68) among 68 cases of pnemocephalus. ${ }^{[7]}$ 
Unlike the systemic venous system, cerebral venous system does not precisely follow the cerebral arterial system. The cortical veins lie superficially and drain to the nearest dural venous sinuses. ${ }^{[8]}$ Dural venous sinuses are connected to each other via various venous plexuses like cervical epidural venous plexus, vertebral venous plexus that surrounds the vertebral artery in the transverse foramen or venous plexus of the hypoglossal canal. The emissary veins provide a venous communication between the dural venous sinuses and the extracranial venous system by passing through foramina in the skull. ${ }^{[9]}$

CVAE is assumed to be resulted from retrograde progression of low weight air bubbles through the subclavian vein into the jugular vein against to the blood flow and then pass into the dural venous sinuses. Besides the air volume, the type of medical procedure and the position of the patient are among other contributing factors. For example, increased intrathoracic pressure during valsalva maneuvers, jugular vein insufficiency and low flow states like in heart failure, as in our case may result in venous air embolism. ${ }^{[3]}$ Venous air embolism can also involve the cerebral arterial vasculature by paradoxical air embolus with the entry of the air from the venous system to the arteries via a right-to-left shunt, such as a patent foramen ovale. ${ }^{[1]}$ In our patient, it seems that after traversing the subclavian vein and reaching the internal jugular vein (IJV), air bubbles migrated on a retrograde course and arrived to the right sigmoid and transverse sinuses. From the IJV, they also migrate through the inferior petrosal sinus to enter the cavernous sinuses and then the ophtalmic veins. Epidural venous air embolism (pneumorrhachis) located in the cervical epidural venous plexus can be explained with its connections to the sigmoid sinus within the skull. This venous plexus also have a connection with the vertebral venous plexus via a venous network at the level of foramen magnum. Epidural venous air embolism has been more frequently described as a complication of epidural anesthetic procedures causing injury of the plexus and resulting in the inadvertent entry of the air into the veins. ${ }^{[10]}$ The air bubbles associated with the venous plexus of the hypoglossal canal in our case could be provided via its relation with the cervical epidural venous plexus, the inferior petrosal sinus and also the IJV. ${ }^{[9]}$ The extracranial air bubbles in the right pterygoid venous plexus and the right superficial temporal vein was thought to be migrated from the dural venous sinuses via emissary veins. In our case, we thought that the emissary vein of Vesalius which is a well known important route for spread of infections to the cavernous sinuses, was responsible for the passage of air from the cavernous sinuses into the pterygoid venous plexus and the superficial temporal vein. ${ }^{[11]}$

The most useful radiological imaging modality to detect intracranial air bubbles is conventional CT scan without contrast. However, as air is absorbed rapidly, it may be only diagnostic in the acute period. Air bubbles appear as extremely dark with Hounsfield unit (HU) of around -1000. Intracranial lipomas have a similar contrast in parenchymal window, but can easily be differentiated by their lower HU (-50 to -100$)$. In our patient, there was a small midline lipoma adjacent to the falx cerebri with HU of average -61 , which has similar contrast with the air within the right transverse sinus (Figure 1a).

The treatment of CVAE consists Trendelenburg positioning to facilitate the return of air bubbles into the central venous system and high concentration (100\%) oxygen to reduce the volume of air in the circulation. Hyperbaric oxygen therapy should be considered for more severe cases. ${ }^{[2]}$ Intravascular volume expansion to prevent further entry of air into the circulation may be considered in appropriate settings. In our patient, with the consideration of her recent and ongoing congestive status, IV hydration was not administered. In addition, avoidance of Valsalva maneuver like coughing or forceful sneezing in order not to increase the intratoracic pressure is recommended. Our patient became symptom free with improvement of the complaints without any deterioration in her clinical status during the follow up at the emergency department.

\section{Conclusion}

CVAE is an unusual but potentially serious complication of various medical procedures. It may occur even after injection of IV fluids at routine peripheral venous lines and result in widespread air bubbles in various locations of the cerebral venous vasculature, as in our case. It should be considered in differential diagnosis of nontraumatic headache and vomitting especially when there is a recent insertion of venous catheters (central or peripheral). CT is gold standard in early diagnosis and management especially during acute phase.

\section{Conflict of Interest}

No conflict of interest.

\section{Author Contributions}

BE: concept, design, analysis, literature search, manuscript writing; KK: design and analysis; AÖA: concept, design and analysis. 


\section{Ethics Approval}

Signed consent was obtained from the patient to publish her data however; ethical approval of the institution was not required.

\section{Funding}

The authors received no financial support for this study.

\section{References}

1. Klein J, Juratli TA, Weise M, Schackert G. A systematic review of the semi-sitting position in neurosurgical patients with patent foramen ovale: how frequent is paradoxical embolism? World Neurosurg 2018;115:196-200.

2. Muth CM, Shank ES. Gas embolism. N Engl J Med 2000;342:476-82.

3. Bothma PA, Schlimp CJ. Retrograde cerebral venous gas embolism: are we missing too many cases? Br J Anaesth 2014;112:401-4.

4. Allioui S, Zaimi S, Sninate S, Abdellaoui M. Air bubbles in the brain: retrograde venous gas embolism in the cavernous sinus. Radiol Case Rep 2020;15:1011-3.
5. Chuang DY, Sundararajan S, Sundararajan VA, Feldman DI, Xiong W. Accidental air embolism. Stroke 2019;50:e183e186.

6. Ploner F, Saltuari L, Marosi MJ, Dolif R, Salsa A. Cerebral air emboli with use of central venous catheter in mobile patient. Lancet 1991;338:1331.

7. Tran P, Reed EJM, Hahn F, Lambrecht JE, McClay JC, Omojola $M F$. Incidence, radiographical features, and proposed mechanism for pneumocephalus from intravenous injection of air. West J Emerg Med 2010;11:180-5.

8. McMinn RMH (editor). Last's anatomy. Revised 9th ed. Chatswood: Elsevier Australia; 2019. p. 741-75.

9. Standring $\mathrm{S}$ (editor). Gray's anatomy: the anatomical basis of clinical practice. 40th ed. London: Churchill Livingstone Elsevier; 2008. p. 584.

10. Souza MP, Magalhães E, Cascudo Ede F, Jogaib MA, Silva MC. Accidental catheterization of epidural venous plexus: tomographic analysis. Rev Bras Anestesiol 2016;66:208-11.

11. Lanzieri CF. The significance of asymmetry of the foramen of Vesalius. AJNR Am J Neuroradiol 1998;9:1201-4.

Correspondence to: Berrin Erok, MD Department of Radiology, University of Health Sciences, Prof. Dr. Cemil Taşçığlu City Hospital, Istanbul, Turkey Phone: +90 5302604150 e-mail: drberrinerok@hotmail.com

Conflict of interest statement: No conflicts declared.

This is an open access article distributed under the terms of the Creative Commons Attribution-NonCommercial-NoDerivs 4.0 Unported (CC BY-NCND4.0) Licence (http://creativecommons.org/licenses/by-nc-nd/4.0/) which permits unrestricted noncommercial use, distribution, and reproduction in any medium, provided the original work is properly cited. How to cite this article: Erok B, Kıbıcı K, Atça AÖ. Retrograde cerebral venous air embolism and the anatomical pathway of air bubbles: a case report. Anatomy 2020;14(3):210-215. 